\title{
How are graphs read? An indication of sequence
}

\author{
RUSSELL W. JONES, JOHN W. WARNER, and CHERIE L. CROSS \\ University of Melbourne, Parkville, Victoria, Australia
}

\begin{abstract}
Graphs are an extremely powerful communicative and analytical tool commonly used in both the behavioral sciences and computing (as well as many other fields). More than 2.2 trillion graphs are published annually, and these graphs are used to communicate a host of often very important information to readers. Yet despite the multitude of applications for which graphs are used, and despite the frequency of their use, little is known about how graphs communicate information or about the cognitive processes that readers use when they read and interpret the information presented within graphs. Insight into the answers to these questions can be obtained through the study of the techniques that people use to read graphs. This paper describes the research methodology and results of an empirical investigation into the viewing order in which readers choose to view the different components of graphs and into the length of time that readers spend studying each of these components.
\end{abstract}

Graphs are communicative tools in which data are encoded in visual images (Lohse, Walker, Biolsi, \& Reuter, 1991 ) for the purposes of communication and/or data analysis. A cursory viewing of television news or a brief browse through a daily newspaper will quickly reveal the importance of graphs as tools for communication, just as a glance at most research summaries prepared by any psychological, social, scientific, medical, or industrial research facility will reveal the importance of graphs as useful tools for data analysis. It has been estimated that more than 2.2 trillion graphs are printed annually (Jones \& Careras, 1996), and many more are produced daily for display on television and computer monitors. The use of graphical software is increasingly widespread, and the increasing accessibility of this software to broader sections of our community (Watson \& Pereira-Mendoza, 1996) portends an even greater importance for graphs as communicative and analytical tools in the future.

Yet despite the enormous number of graphs that are produced, their increasingly widespread use throughout all facets of our society (Feeney, 1994), and their involvement in the communication of information within many fields (including, in addition to computing and psychology, all the other sciences, humanities, medicine, the media, private industry, the military, government, and educational organizations), there exists a paucity of empirical research into the ways in which graphs communicate information to readers. Recognition of this dearth of empirical research is not new; as early as 1874, Mayr drew attention to the need for more empirical research, as did Croxton and Stryker in 1927. More recently, Cleveland (1985); Cleveland and McGill (1986); Dempsey, Fisher, and Marousky (1994); Jones and Careras (1996); Morley and

The authors would like to thank Diane Schiano, Darrell Butler, and an additional (anonymous) reviewer for their comments on an earlier draft of this article. Correspondence should be addressed to R. W. Jones, DSME, University of Melbourne, Parkville, Victoria 3052, Australia (r.jones@edfac.unimelb.edu.au).
Adams (1991); and Tufte (1983) have also called for more empirical research. Most recently, the call for greater empirical research has been accompanied by a concomitant call for greater understanding about how people read graphs (e.g., Jackson, Edwards \& Berger, 1993; Lohse et al., 1991; Martin, Chiu, \& Dailey, 1990; Pinker, 1990; Rangecroft, 1991: Tversky \& Schiano, 1989; Watson \& Pereira-Mendoza, 1996).

In particular, little is known about how graphs communicate information to their readers or about the cognitive processes that readers use to read and interpret the information presented in graphs. However, some valuable studies have been published. Wickens and Andre (1990) have reported the results of an experiment in which they compared three indicators of aircraft stall danger either as a bar graph or as combined features in an object display. These researchers found that, relative to the bar graph, the object display disrupted focused attention but improved information integration. Sanderson, Flach, Buttigieg, and Casey (1989) also compared the relative superiority of bar graphs and object displays. They have reported two studies in which, in contrast to the findings of Wickens and Andre (1990), they found bar graphs to be superior to object displays if the bar graph had a strong emergent feature that mapped directly onto the task. It is likely that such a "strong emergent feature" caused greater cognitive attention to be paid to the variable encoded within the feature, thereby enhancing the reader's ability to cognitively process the variable. Also to be considered as an influence on cognition is the type of tasks that readers can perform while reading graphs. The cognitive processing of many graphical displays may involve the reader's consideration either of a relationship among several variables (often called integrated tasks) or of specific values of individual variables (often called focused tasks). Bennett and Flach (1992) examined the interaction between visual features within graphical displays and processing capabilities from the perspective of basic research on attention and object perception. These authors were able to conclude that 
it may be possible for graph designers to design graphs requiring both integrated and focused tasks with a realistic expectation that readers can successfully master both tasks.

In addition to benefiting from examinations of task performance as a function of graph characteristics, as in the prior studies, graph design should benefit from an understanding of the techniques that people use to read graphs. Certainly insight into the cognitive processing that occurs while people interpret information presented in graphs can be obtained through study of the techniques that people use to read graphs. Of particular interest for the study reported in this article was the viewing order in which a reader chooses to look at the various components within a graph and the length of time that a reader spends studying each component. Answers to these questions provide clues about the cognitive strategies that readers use when they read graphs. In addition, such answers may provide invaluable guidance to those involved in the design of graphs.

\section{STATEMENT OF PURPOSE}

The research presented here describes a new software tool and an empirical study of (1) the order in which readers choose to view the components of a graph, and (2) the measurement of the amount of time that readers take to study each of these components. The purpose of this research was to increase our knowledge of how readers view the different components that constitute graphs. Specifically, we sought to answer the following questions: Is there a statistically significant order in which the parts of a graph tend to be viewed by readers and, if so, what is this order? How much time do readers spend in studying each graphical component, and is there a statistically significant difference between the amounts of time that readers spend in viewing each component?

\section{METHOD}

\section{Software Tool}

A computer program, or software tool, was developed to conduct this investigation. The software tool was written in Visual Basic, Version 4.0 (Microsoft, 1995), and run in a Windows 95 environment. This tool, Graphing Components (Warner \& Jones, 1997), was designed to collect both survey and experimental data. Graphing Components presents subjects with a series of graphs. When each graph is initially displayed, it is covered by a series of seven buttons. These buttons cover each of the seven components of a graph; title, data region (or body), legend, $x$-axis, $y$-axis, $x$-axis label, and $y$-axis label. Each button is labeled with the name of the graph component that it covers (i.e., title, body, legend, etc.). For a subject to view the portion of a graph that is covered by each button, the subject must use the computer mouse to "click" on the button. Once "clicked," the button disappears, revealing the graph component. Subjects study this component until they are ready to uncover another component, whereupon they click on another button to uncover another portion of the graph. Subjects thus progressively uncover the components of the graph until the entire graph is revealed (see Figure 1). As subjects work through each task, the computer is programmed to record the order in which each graph component is uncovered; the length of time that subjects spend studying each component; and each subject's answer to a stimulus question (the program has been designed to record both numerical and textual information).

The seven components of a graph that are used in this software were selected during initial experimental design. At this stage, we asked subjects a question, the answer to which required them to study a graph. While they were studying the graph, they were required to state what parts of the graph they were looking at. This exercise revealed a general consensus among subjects that they tended to view seven discrete components; title, data region (or body), legend, $x$-axis, $y$-axis, $x$-axis label, and $y$ axis label.

Graphing Components is designed to guide subjects through the entire program without human supervision. This research tool has been published on the Web and is

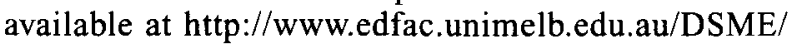
research/

This software gathers its data by constructing a string that comprises text codes for each event associated with each graph. For events for which time is to be recorded, a call is made to the Windows API millisecond timer, and the time is included in the string. Textual answers to questions are filtered to ensure that there are no characters in the subject's text responses that would upset the process of translating this string into legal commadelimited input. Such input is useful for importing into data-handling software packages such as an Excel spreadsheet or a statistical package. The string is written to a file after each experimental subject has completed the tasks within Graphing Components. These strings accumulate in a file called results.dat.

\section{Windows of Opportunity}

During initial experimental design, much consideration was given to deciding whether to write the software to either progressively uncover the graph or restrict subjects" viewing to only one viewing "window" at a time, thereby permitting subjects to view only one component of the graph at any time. For the purposes of the investigation reported here, it was decided that progressively uncovering a graph would allow the opportunity to gather information about how long subjects spent studying the graph. Hence, the program Graphics Components was written to enable subjects to gradually uncover each graph and to ultimately view graphs in their entirety. It may be argued, however, that useful information would also be gathered with a software tool that restricted subjects' viewing to a single graph component at any time. To address this argument, a second software tool has been designed, entitled Windows of Opportunity. It is similar 

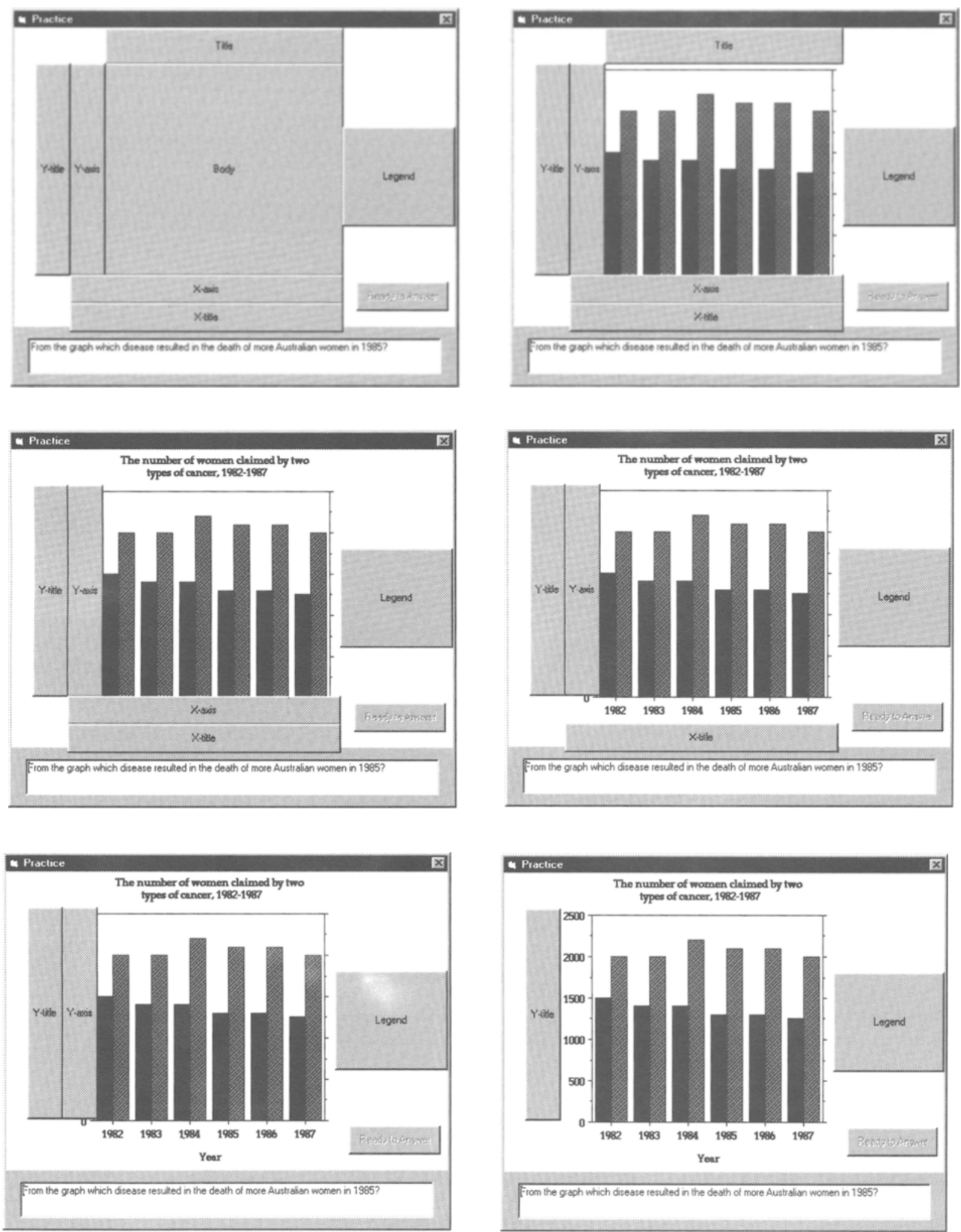

Figure 1 continued on next page. 

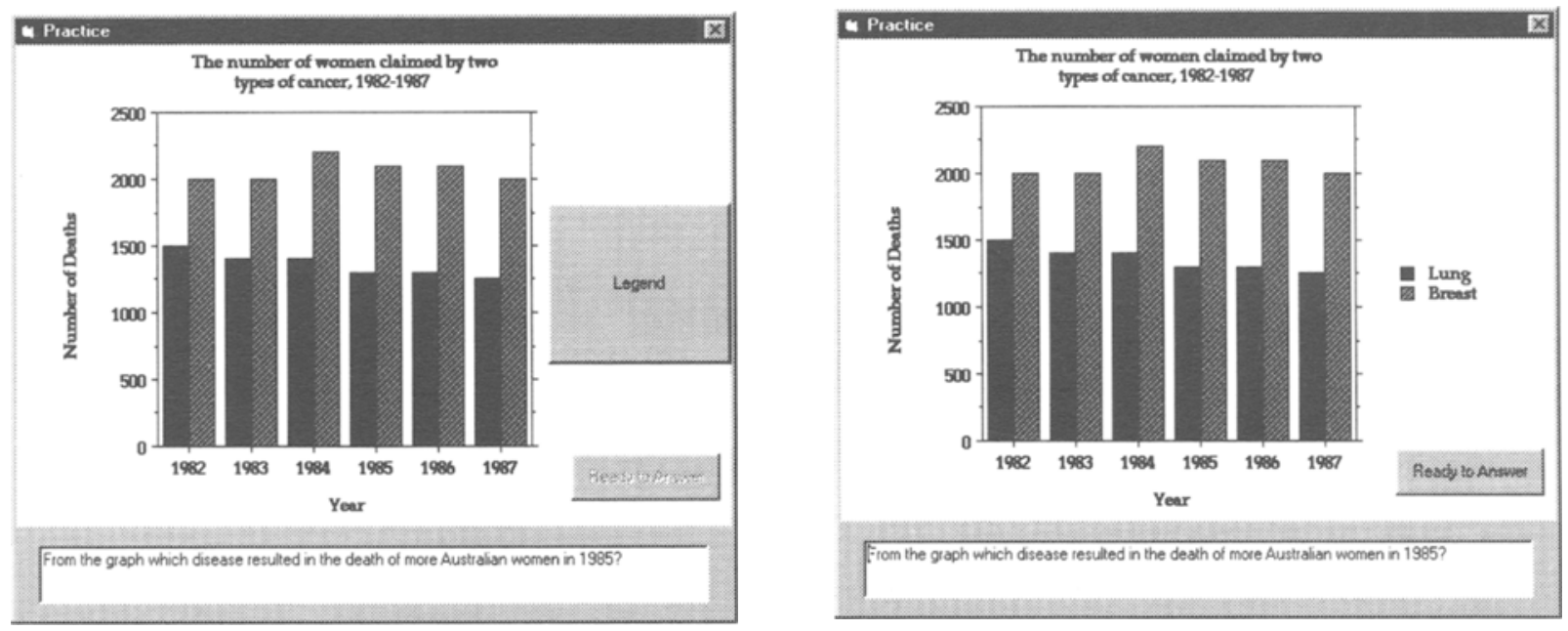

Figure 1. A sequence showing a covered, progressively uncovered and fully uncovered graph.

to Graphics Components, except that subjects are able to view only one component at a time. When each graph is initially displayed, it is covered by seven buttons. When a subject "clicks" on any button, the button disappears, offering the subject a window of opportunity to view a specific component. When a subject has completed study of the graph component, he/she clicks on the button covering another component, whereupon this component is revealed and the previous component is re-covered. Windows of Opportunity has been published on the Web and is also available at http:/www.edfac.unimelb.edu.au/ DSME/research/

\section{Experimental Stimuli}

Two forms of stimuli were included within Graphing Components: a series of questions and a matching series of graphs. Each question was administered along with the graph that contained the answer to the question. Subjects were instructed to answer each question by using the information embedded within the accompanying graph. All of the stimuli were displayed on a computer monitor. The questions were displayed on the monitor prior to the accompanying graph, and, when the graph was displayed, the accompanying question was also displayed beneath the graph to serve as a reminder to the subjects. Thus subjects were able to concentrate on reading and interpreting the graph rather than having to concentrate on memorizing the question.

The questions were phrased so that all seven components of the graph needed to be uncovered before subjects had sufficient information to answer each question. And, once the graph was uncovered, the subjects were able to record their answer to the question by clicking on a button labeled "Ready to Answer," whereupon the graph disappeared and a text box appeared on the monitor. The disappearance of the graph from the monitor prior to the subjects' being able to record their answers ensured that the subjects completed their study of the graphs prior to answering the questions (this was important because an important part of this experiment involved calculating the length of time that subjects spent in studying each graph).

To maximize consistency between graphs, all graphs were identical in terms of size and comprised approximately the same number of pieces of information (see Figure 2). For example, the data region in each graph contained 12 discrete pieces of data, two variables were encoded within each graph, all graph legends contained information pertaining to two variables, and so forth.

Prior to commencing the experimental portion of the program, each subject was given a series of three practice tasks identical to those within the experimental tasks. The experimental portion consisted of six tasks. Because bar charts, line charts, and scatterplots are the three most frequently used graph formats (Lewandowsky \& Spence, 1989), one third of the tasks contained graphs with each of these formats. Because most graphs are typically drawn in two dimensions, rather than three (Tall, 1992), only two-dimensional graphs were used. To avoid any ordering effects that might arise from the sequence with which graphs were presented to subjects, the computer was programmed to randomly select graphs for presentation to subjects. The computer was also programmed to ensure that when a graph was selected, the matching question relating to the graph was also selected.

Although the program was designed to operate independently of the need for human supervision, a research assistant remained in the room while subjects worked through the program. This was found to be desirable, because the presence of a research assistant during the piloting of the experimental procedure increased the value that subjects appeared to place on the completion of the tasks.

The program was designed to ask and record typical demographic and background information from subjects (e.g., age and gender). In an effort to determine the extent 

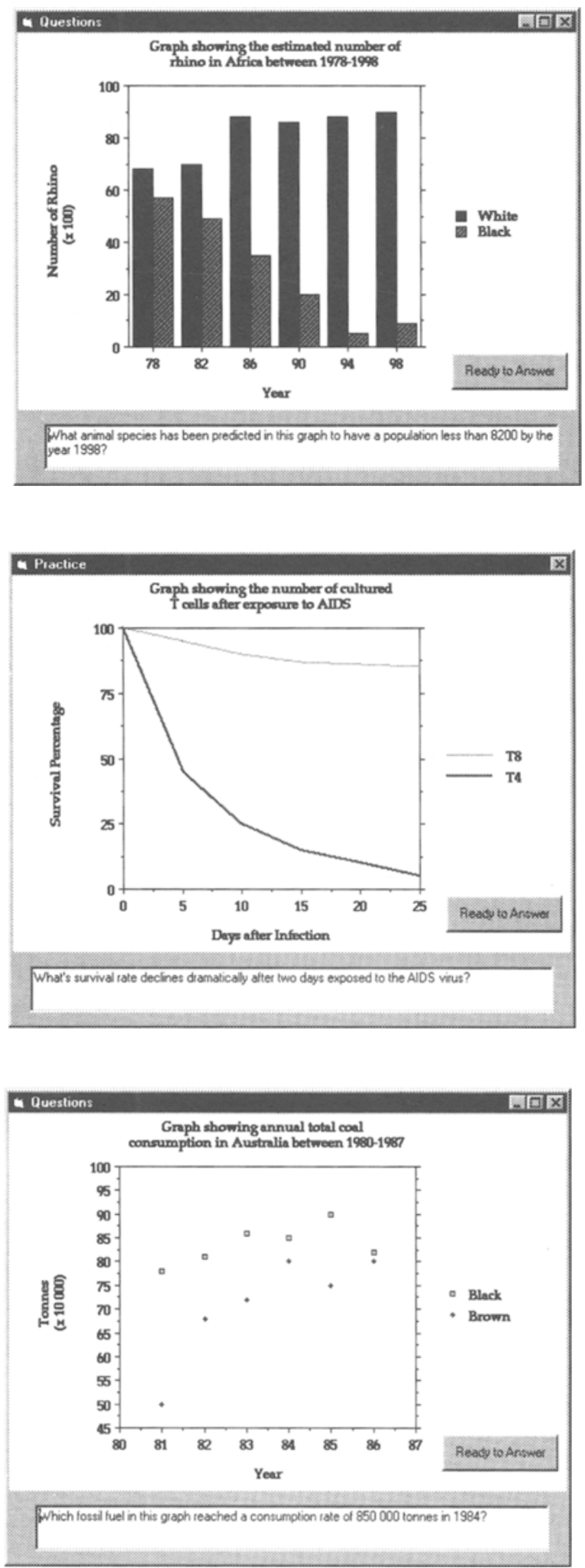

Figure 2. A sample bar chart, line chart, and scatterplot. 
Table 1

Number of Courses Subjects Reported Having Taken in Statistics, Graphic Arts, and Visual Arts

\begin{tabular}{lcccc}
\hline & \multicolumn{5}{c}{ Number of Courses } \\
\cline { 2 - 5 } \multicolumn{1}{c}{ Subject } & None & One & Two & Three or More \\
\hline Statistics & 9 & 45 & - & 3 \\
Graphic Arts & 47 & 7 & 1 & 1 \\
Visual Arts & 52 & 4 & - & 1 \\
\hline
\end{tabular}

of subject familiarity with graphs and subject familiarity with visually presented stimuli (which may have an effect on a subject's ability to read graphs), the program also asked subjects to report their experience with graphing, statistics, visual arts, and graphical arts on a four-point Likert scale.

\section{Subjects}

The task was administered to 57 university students in their 4th year of college-level study. Twenty-five were male, and 32 were female. Their ages ranged from 20 to 35 , the median being 21 . Eight percent of the students reported "no experience" of reading graphs, 57 percent reported "some experience," 23 percent reported "moderate experience," and $17 \%$ reported "extensive experience." An estimate of subject experience in other areas that might influence subject ability to read and interpret graphs was obtained by asking the subjects to report the number of courses that they had taken in statistics, graphic arts, and visual arts. These are reported in Table 1.

\section{RESULTS AND DISCUSSION}

\section{Component Viewing Order}

The order in which subjects chose to view the seven graphing components is summarized in Table 2 . The first column specifies the graph component. The second through eighth columns record the number of occasions when each of the graph components was chosen first, second, third, fourth, fifth, sixth, or seventh. For example, the 223 in the first row of the second column indicates that the title was selected first by subjects on 223 out of the possible 342 occasions ( 6 experimental graphs $\times 57$ subjects $=342$ occasions), and the 126 in the seventh row of

Table 2

General Tendency to Select Title First, the Data Region and Legend Toward the Beginning of the Task or Toward the End, and the Axes and Labels From Left to Right and Top to Bottom

\begin{tabular}{lrrrcccc} 
& \multicolumn{7}{c}{ Order of Selection } \\
\cline { 2 - 8 } Component & First & Second & Third & Fourth & Fifth & Sixth & Seventh \\
\hline Title & 223 & 16 & 14 & 13 & 19 & 12 & 45 \\
Data region & 86 & 76 & 31 & 14 & 19 & 58 & 58 \\
$y$-label & 6 & 75 & 66 & 61 & 48 & 41 & 45 \\
$y$-axis & 9 & 57 & 84 & 86 & 52 & 49 & 5 \\
$x$-axis & 7 & 31 & 70 & 85 & 84 & 55 & 10 \\
$x$-label & 8 & 14 & 49 & 50 & 88 & 80 & 53 \\
Legend & 3 & 73 & 28 & 33 & 32 & 47 & 126 \\
\hline
\end{tabular}

the eighth column indicates that the legend was selected last on 126 occasions. Chi-square analyses revealed that graph components were selected in a statistically significant order $\left[\chi^{2}(36, N=342)=48.86, p<.01\right]$.

Title. The title was viewed first during the majority of trials. Subjects may have chosen this component of the graph first because of a genuine desire to view the title first in order to determine what the graph was "about," or because of a force of habit established through years of reading experience (whereby people start reading at the top of a page), or both. It would be informative to repeat this experiment with subjects from cultures that traditionally begin reading a page from the bottom rather than the top. An alternative would be to change the location of the title within a graph, yet this would change the structure of the graph from that typically used by graph publishers.

Data region. The data region had a bimodal selection pattern, tending to be selected first through second or sixth through seventh. This is indicative of subjects' possibly seeking to either (1) view the data early in their study of the graph in order to begin to analyze the graph in relation to the information contained in each of the other components, or (2) cognitively assimilate the other components of the graph before viewing the data.

Labels and axis. There was a tendency for the $y$-label to be selected second through fourth, and for the $y$-axis, second through fifth. Similarly, there was a tendency for the $x$-axis to be selected third through sixth, and for the $x$-label, fourth through seventh. Overall, these data suggest that components relating to the $y$-axis tended to be viewed before components relating to the $x$-axis. This may be related to viewing habits obtained through the reading of normal text, whereby readers tend to read a page from left to right and from top to bottom.

Legend. The legend had a bimodal selection pattern, tending to be selected second or last. Selection second would seem to make sense for subjects who chose to view the data region first, because the legend was closely linked to the data. The legend was selected last on a high proportion of trials, however, and this may be because subjects typically thought the legend to be least important or because of the location of the legend at the extreme right edge of the graph.

A possible sequence. Although the findings reported above are far from conclusive, it is possible to derive a general pattern from them, as can be seen in Figure 3.

A typical reader (if such a person exists) might view the title first in order to determine the graph topic; move on to the data region in order to view the major substance of the graph (i.e., the data); then, once these substantive and usually significant pieces of information had been obtained and while they were being cognitively processed within working memory, might fall into reading habits to view the remainder of the graph, working from left to right and down to view the $y$-label, $y$-axis, $x$-axis, and $x$-label. The legend might be viewed last, because, 


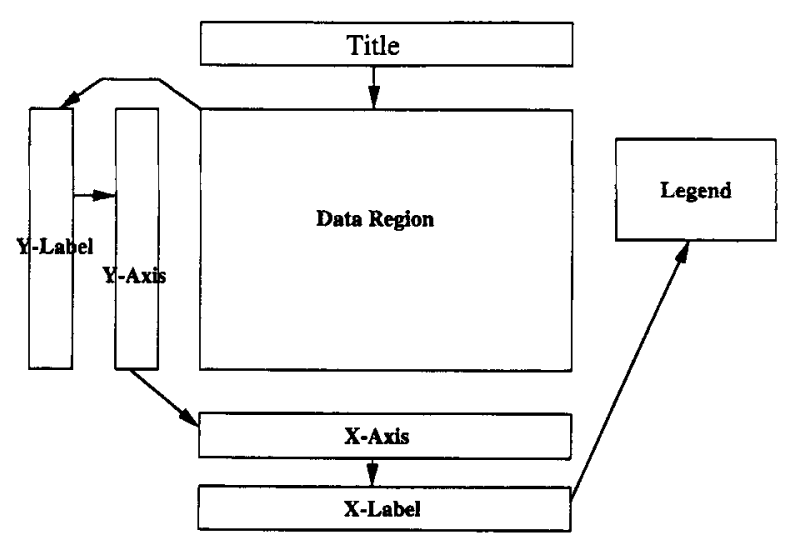

Figure 3. A possible viewing route used by subjects as they read a graph.

for some yet to be determined reason, it might be cognitively best viewed last, or it might simply be cognitively inefficient for the eyes to move entirely from the left portion of the graph after viewing the $y$-axis to the extreme right of the graph to view the legend and then back to the $y$-axis. Admittedly the preceding suggestions represent a large step, given the evidence reported earlier; yet such an analysis offers a tantalizing glimpse into the rationales that determine how people read graphs. Certainly this is an area worth further study.

\section{Time Spent Viewing Each Component}

An analysis of variance (ANOVA) was used to establish any differences among the lengths of time that subjects spent studying the seven graph components. The results of the ANOVA are presented in Table 3. Examination of this table readily reveals statistically significant differences in the length of time that subjects spent in viewing the graph components. To identify the precise source of these differences, a series of post hoc Bonferroni analyses were conducted. The results are summarized in Table 4. The times in Table 4, in milliseconds, represent the mean differences in viewing time between graph components. Significant differences are indicated by an asterisk.

A statistically significant difference was found between the time that subjects spent viewing the graph title and the time spent viewing all other components of the graph except the legend. Similarly, a statistically significant difference was found between the time that subjects spent viewing the graph legend and the time spent viewing all other components of the graph except the title. Subjects spent significantly more time viewing the title $(4,375 \mathrm{msec})$ and legend $(4,384 \mathrm{msec})$ than they did viewing any other component. The time that subjects spent viewing the data region $(3,216 \mathrm{msec})$ was significantly less than the time spent studying either the title or the legend, yet it was significantly more than the time spent studying the $x$-label. No statistically significant differences were found between the lengths of the time that subjects viewed the $x$-axis $(2,730 \mathrm{msec}), x$-label $(2,270 \mathrm{msec}), y$-axis $(2,480 \mathrm{msec})$, or $y$-label $(2,745 \mathrm{msec})$.
It seems reasonable to expect subjects to spend the most time studying components that contain the most information and the least time studying components that contain the least information. This would explain the brief viewing times associated with the $x$ - and $y$-axes and labels (small differences between the viewing times for these components can be explained by factors such as its taking slightly longer for subjects to read the vertical $y$ label than to read the horizontal $x$-label). Hence, because the title, legend, and data region contain much more information than that found associated with the axes, subjects should spend more time viewing these components. Indeed, the results show that subjects spend most time viewing these components. However, given this rationale, it would seem logical to expect subjects to spend the most time viewing the data region, because this is the component containing the most information. Yet Table 4 shows that subjects spent an average of $941 \mathrm{msec}$ less viewing the data region than they did the title, and $1,168 \mathrm{msec}$ less viewing the data region than they did the legend. Discussions with subjects following completion of the tasks within Graphing Components revealed that many subjects tended to briefly glance at the data region to obtain a mental picture of the major features and trends of the data, with the intention to return to more careful study of the data once the entire graph was revealed. This strategy seems logical and was consistent with the expectations of the authors. If subjects did indeed return their attention back to the data region after the graph was entirely exposed in order to study the data more closely in relation to information contained in the rest of the graph, there should have been a substantial time interval between the moment at which the graph was entirely exposed and the moment at which a subject indicated he/ she was ready to answer the question. This is indeed what happened, and subjects were found to spend a mean of

Table 3

Results of an ANOVA to Establish Any Differences in the Times Students Spent Studying Each Graph Component

\begin{tabular}{lcrccc}
\hline \multicolumn{1}{c}{ Time } & $S S$ & $d f$ & $M S$ & $F$ & $p<$ \\
\hline Between groups & $1.65 E+09$ & 6 & $2.75 E+08$ & 13.464 & .00 \\
Within groups & $6.27 E+10$ & 3,071 & $20,418,366$ & & \\
Total & $6.44 E+10$ & 3,077 & & & \\
\hline
\end{tabular}

Note-SS = sum of squares.

Table 4

Results of Bonferroni Analyses Showing That Subjects Spent Significantly More Time Initially Viewing the Legend and Title Than the Data Region, and Significantly Less Time Viewing the Axes and Labels Than the Data Region, Title, or Legend Data Region Legend Title $x$-axis $x$-label $y$-axis Legend $-1,168^{+}$ $\begin{array}{lccc}\text { Title } & -941^{*} & 227 & \\ x \text {-axis } & 485 & 1,654^{\dagger} & 1,427^{\star}\end{array}$

$x$-label $\quad 946^{*} \quad 2,114^{\ddagger} \quad 1,887^{\ddagger}$

$\begin{array}{llllll}y \text {-axis } & 736 & 1,904^{\ddagger} & 1,677^{\ddagger} & 250 & -210\end{array}$

$\begin{array}{lllllll}y \text {-label } & 470 & 1,639 \ddagger & 1,412^{\ddagger} & -15 & -475 & -265\end{array}$

${ }^{*} p<.05 . \quad{ }^{\dagger} p<.01 . \quad{ }^{\ddagger} p<.001$. 
28,615 msec studying the graph "as a whole" before indicating that they were ready to answer the question. It seems reasonable to expect that a substantial portion of this time was spent in studying the data region.

In the study reported here, the use of Graphing Components (which eventually permits subjects to view graphs in their entirety), rather than Windows of Opportunity, goes part way toward addressing issues relating to gestalt. However, the fact that subjects were unable to view graphs in their entirety throughout the tasks may remain an important limitation of the present study. Indeed the issue of gestalt is a valid concern for any study in which the visual images available to subjects are restricted in any way.

\section{CONCLUSION}

The aim of this research was to increase our knowledge about how readers view the different components that constitute a graph. The results have both practical and theoretical implications. The practical implications apply to graph authors, graphics designers, and those involved in the presentation of graphical displays. For example, graph authors should make the content and appearance of graph titles eye-catching and appealing to readers, because this is the component of a graph most readers read first. If the content and/or appearance of a graph title is catching, this would seem to maximize the likelihood that a reader will read the title, and, if the reader finds the content of the title appealing, this would seem to maximize the likelihood that a reader skimming through a publication will continue to read the remainder of the graph and possibly any adjoining text. The theoretical implications include knowledge of the order by which readers study each component of a graph. This provides insight into the cognitive strategies that readers use to read graphs. Similarly, the time that readers spend viewing each component provides cognitive psychologists, and others interested in the way people read graphs, with clues about the depth and possible nature of the cognitive processing that readers use as they seek to interpret the enormous amount of data that are encoded within the multitude of graphs produced and published every day.

\section{REFERENCES}

BenNetT, J. M., \& FLACH, J. M. (1992), Graphical displays: Implications for divided attention, focused attention, and problem solving. Human Factors, 34, 513-533.

CLEVELAND, W. S. (1985). The elements of graphing data. Monterey, CA: Wadsworth
Cleveland, W. S., \& McGill, R. (1986). An experiment in graphical perception. International Journal of Man-Machine Studies, 25, 491500.

Croxton, F. E., \& Stryker, R. E. (1927). Bar charts versus circle diagrams. Journal of the American Statistical Association, 22, 473-482.

DempSey, J. V., Fisher, S., \& Marousky, R. (1994, April). Preference and use of information in instructional graphs. Paper presented at the annual conference of the National Consortium for Instruction and Cognition, New Orleans.

FeENEY, M. (1994, March 11). Beyond the voodoo stick: If a picture is worth a thousand words, how many words is an information graphic worth? The Boston Globe Magazine, pp. 10-18.

JACKSON, D. F., EDWARDS, B. J., \& BERGER, C. F. (1993). Teaching the design and interpretations of graphs through computer-aided graphical data analysis. Journal of Research in Science Teaching, 30, 483501 .

Jones, R. W., \& Careras, I. E. (1996). The empirical investigation of factors affecting graphical visualization. Behavior Research Methods, Instruments, \& Computers, 28, 265-269.

LEWANDOWSKY, S., \& SPENCE, I. (1989). The perception of statistical graphs. Sociological Methods \& Research, 18, 200-241.

Lohse, G., Walker, N., Biolsi, K., \& Reuter, H. (1991). Classifying graphical information. Behaviour \& Information Technology, 10, 419-436.

Martin, J., Chiu, M-H., \& Dailey, A. (1990). Graphing in the second grade. The Computing Teacher, 18, 28-32.

MAYR, G. VON (1874). Gutrachten über die Anwendung der graphischen und geographischen Methoden in der Statistik [Opinions on the use of graphical and geographical methods in statistics]. Munich: J. Gotteswinter \& Mossl.

Microsoft CoRPoration (1995). Visual Basic (Professional Version 4.0). Redmond, WA: Author.

MorleY, S. R., \& AdAMs, M. (1991). Graphical analysis of single case time series data. British Journal of Clinical Psychology, 30, 97-115.

PINKER, S. (1990). A theory of graph comprehension. In R. Freedle (Ed.), Artificial intelligence and the future of testing (pp. 73-126). Hillsdale, NJ: Erlbaum.

RANGECROFT, M. (1991). Graphwork, developing a progression: A diversity of graphs. Teaching Statistics, 13, 90-92.

Sanderson, P. M., Flach, J. M., Buttigieg, M. A., \& Casey, E. J. (1989). Object displays do not always support better integrated task performance. Human Factors, 31, 183-198.

TALL, D. (1992). Visualizing differentials in two and three dimensions. Teaching Mathematics \& Its Applications, 11, 1-7.

TUFTE, E. R. (1983). The visual display of quantitative information. Cheshire, CT: Graphics Press.

Tversky, B., \& Schiano, D. J. (1989). Perceptual and conceptual factors in distortions in memory for graphs and maps. Journal of Experimental Psychology: General, 118, 387-398.

WARNER, J. W., \& JONES, R. W. (1997). Graphing components [Computer program]. Melbourne: University of Melbourne.

Watson, J., \& Pereira-Mendoza, L. (1996). Reading and predicting from bar graphs. Australian Journal of Language \& Literacy, 19, 244-258.

WiCKens, C. D., \& ANDre, A. D. (1990). Proximity compatibility and information display: Effects of color, space, and objectiveness on information integration. Human Factors, 32, 61-77.

(Manuscript received October 21, 1997; revision accepted for publication March 6,1998.) 